\title{
High-Order Approximation to Two-Level Systems with Quasiresonant Control
}

\author{
Lin Wang $\mathbb{D}^{1}$ and Jian $\mathrm{Zu} \mathbb{1}^{2}$ \\ ${ }^{1}$ School of Mathematics and Statistics, Changchun University of Technology, Changchun 130012, China \\ ${ }^{2}$ School of Mathematics and Statistics, \& Center for Mathematics and Interdisciplinary Sciences, Northeast Normal University, \\ Changchun 130024, China
}

Correspondence should be addressed to Jian Zu; zuj100@nenu.edu.cn

Received 11 April 2020; Revised 21 July 2020; Accepted 29 July 2020; Published 6 October 2020

Academic Editor: Maria L. Gandarias

Copyright (c) 2020 Lin Wang and Jian Zu. This is an open access article distributed under the Creative Commons Attribution License, which permits unrestricted use, distribution, and reproduction in any medium, provided the original work is properly cited.

In this paper, we focus on high-order approximate solutions to two-level systems with quasi-resonant control. Firstly, we develop a high-order renormalization group (RG) method for Schrödinger equations. By this method, we get the high-order RG approximate solution in both resonance case and out of resonance case directly. Secondly, we introduce a time transformation to avoid the invalid expansion and get the high-order RG approximate solution in near resonance case. Finally, some numerical simulations are presented to illustrate the effectiveness of our RG method. We aim to provide a mathematically rigorous framework for mathematicians and physicists to analyze the high-order approximate solutions of quasi-resonant control problems.

\section{Introduction}

The dynamics of a two-level system interacting with a weak electromagnetic field is a relevant field of study in quantum optics. Scientists have been working to search for a satisfactory long-time approximation for such systems. Rotating wave approximation (RWA), which neglects the fast oscillating terms, is the most commonly used method for physicists (see [1]). Amazingly, slow oscillating terms are often removed by RWA in some models. For example, $2 \cos \theta=$ $e^{i \theta}+e^{-i \theta}=e^{i \theta}\left(1+e^{-2 i \theta}\right) \approx e^{i \theta}$ in RWA, where the neglected term $e^{-i \theta}$ is only two times speed away from $e^{i \theta}$ (see [2]). Is it fast enough to be neglected? Despite RWA is a "rude" method, it usually gives a good approximation for some important models in quantum optics. However, if we can construct a more reliable approximation is an important issue in quantum optics. Scientists are always looking for the methods without RWA (for example [3-5]). In this paper, we aim to provide a mathematically rigorous frame- work for mathematicians and physicists to get satisfactory long-time approximate solutions for such systems.

Suppose that the open quantum systems are composed of a stable states $|g\rangle$ and an excited state $|e\rangle$ with $\langle g, e\rangle=0$. The free Hamiltonian $H_{0}$ and the coupling Hamiltonian $H_{1}$ are defined by

$$
\begin{aligned}
& H_{0}=\lambda_{g}|g\rangle\left\langle g\left|+\lambda_{e}\right| e\right\rangle\langle e|, \\
& H_{1}=|g\rangle\langle e|+| e\rangle\langle g|,
\end{aligned}
$$

where $\lambda_{g}$ and $\lambda_{e}$ are the eigenvalues of the free Hamiltonian $H_{0}$ with respect to $|g\rangle$ and $|e\rangle$, respectively. The evolution of the state of a quantum system with quasi-resonant control can be described by the following Schrödinger equations

$$
i \frac{d}{d t} \psi=\left(H_{0}+\mathscr{U}(t ; \varepsilon) H_{1}\right) \psi
$$

where the wave function $\psi$ belongs to a finite-dimensional 
Hilbert space, and the quasi-resonant control $\mathcal{U}(t ; \varepsilon)$ is a weak electromagnetic field control. Here, the quasiresonant control includes the resonance case, near resonance case, and out of resonance case.

Rotating wave approximation is an effective method to deal with the quantum system with resonant control (see [6]). However, to our knowledge, it is not clear how to get the high-order approximate solutions rigorously for the quasi-resonant control. The renormalization group (RG) method proposed by Chen, Goldenfeld and Oono in $[7,8]$ is a unified asymptotic analysis tool which reduces a singular perturbation problem to a more simple equation called the RG equation. Ziane in [9], DeVille et al. in [10], and Chiba in [11] gave the error estimate of RG approximate solutions rigorously. Chiba in [11-13] defined the high-order RG equation and the RG transformation to improve error estimate. The RG method has been further developed in [14-18].

In the present paper, we develop a high-order RG method for Schrödinger equations with quasi-resonant control. Compared to the pioneering work in [12], we adjust the construction of the $m$-order RG approximate solution by getting rid of the $m$-order oscillation part, which is more reasonable to give an explicit form. Our proof is concise and rigorous. The selection of integral constant in our method is also the most suitable for numerical calculation. As a direct application, we apply the high-order RG method to two-level systems in and out of resonance. The numerical simulation shows that the first-order RG approximate solution is usually good enough in the case of resonance, but the second-order RG approximate solution plays a key role in the two-level systems out of resonance.

The dynamic behavior of two-level systems near resonance is the most concerned problem, which is also the original intention of developing the high-order RG method. On the one hand, we need to find out whether the approximate solutions of the two-level systems near resonance have a consistent representation when there are more than one “»" relationships in a physics article; on the other hand, we hope to find out how to avoid an invalid expansion to appear in the case of near resonance. Here, we call the expansion with small parameters in coefficients the invalid expansion. This paper introduces a time transformation technique to avoid the invalid expansion near resonance. Near resonance problems with two-scale case, high-order near resonant case, and high-order weak driving case are discussed carefully. The numerical simulations are presented to illustrate that the first-order RG approximate solution is usually good enough in the high-order near resonant case, but the second-order RG approximate solution plays a fundamental role in the two-scale case and high-order weak driving case.

This paper is organized as follows. In Section 2, we develop a high-order RG method for Schrödinger equations with quasi-resonant control. In Section 3, high-order RG approximate solutions both in and out of resonance are presented. In Section 4, we introduce a time transformation technique to avoid the invalid expansion near resonance. High-order RG approximate solutions in several types of near resonance are discussed in detail.

\section{Renormalization Group Method}

In this section, we generalize the high-order RG method in [12] to Schrödinger equations

$$
\left(1-\varepsilon^{p} \kappa\right) \cdot i \frac{d}{d t} \psi=H_{0} \psi+\varepsilon^{q} G(t) \psi, \quad \psi \in \mathbb{C}^{n}, 0<\varepsilon \ll 1,
$$

where $H_{0}$ is a real diagonal matrix with different eigenvalues, $G(t)$ is a continuous quasiperiodic Hermitian matrix, $p, q \epsilon$ $\mathbb{N}^{+}$is the order of near resonance and weak drive, respectively, and $\kappa>0$ is the detuning parameter. Equation (3) can be rewritten as

$$
\frac{d}{d t} \psi=-i H_{0} \psi+\varepsilon G_{1}(t) \psi+\cdots+\varepsilon^{k} G_{k}(t) \psi+\cdots
$$

where $G_{k}(t)$ is the quasiperiodic skew-Hermitian matrix with the same quasiperiodic frequency. Put $\psi=\psi_{0}+\varepsilon \psi_{1}+\varepsilon^{2} \psi_{2}$ $+\cdots$. Equating the coefficients of each $\varepsilon^{i}$, we have

$$
\begin{aligned}
& \varepsilon^{0}: \frac{d}{d t} \psi_{0}+i H_{0} \psi_{0}=0 \\
& \varepsilon^{k}: \frac{d}{d t} \psi_{k}+i H_{0} \psi_{k}=\sum_{j=0}^{k-1} G_{k-j}(t) \psi_{j}, \quad k \geq 1 .
\end{aligned}
$$

Define the resonant part and oscillating part by

$$
\begin{aligned}
R_{k} \triangleq & \lim _{t \rightarrow \infty} \frac{1}{t} \int_{0}^{t}\left(e^{i H_{0} s} G_{k}(s) e^{-i H_{0} s}+\sum_{j=1}^{k-1} e^{i H_{0} s} G_{k-j}(s) h_{s}^{(j)}\right. \\
& \left.-\sum_{j=1}^{k-1} e^{i H_{0} s} h_{s}^{(j)} R_{k-j}\right) d s, \\
h_{t}^{(k)} \triangleq & e^{-i H_{0} t} \int_{0}^{t}\left(e^{i H_{0} s} G_{k}(s) e^{-i H_{0} s}+\sum_{j=1}^{k-1} e^{i H_{0} s} G_{k-j}(s) h_{s}^{(j)}\right. \\
& \left.-\sum_{j=1}^{k-1} e^{i H_{0} s} h_{s}^{(j)} R_{k-j}-R_{k}\right) d s .
\end{aligned}
$$

Assumption 1. Suppose that $R_{k}, e^{i H_{0} t} h_{t}^{(k)}$ appearing in the finite step RG process are quasiperiodic skew-Hermitian matrices, whose Fourier exponent has no accumulation points.

Define the $m$ th-order RG equation

$$
\frac{d W(t)}{d t}=\varepsilon R_{1} W(t)+\varepsilon^{2} R_{2} W(t)+\cdots \varepsilon^{m} R_{m} W(t), \quad W(t) \in \mathbb{C}^{n},
$$

and the $m$ th-order RG transformation

$$
\alpha_{t}^{(m)}(W(t)) \triangleq e^{-i H_{0} t} W(t)+\varepsilon h_{t}^{(1)} W(t)+\cdots+\varepsilon^{m} h_{t}^{(m)} W(t) .
$$

Let $\psi(t)$ is the solution of (3) with initial value $\psi(0)=\xi$. 
Then $\psi_{\text {app }}(t) \triangleq \alpha_{t}^{(m)}(W(t))-\varepsilon^{m} h_{t}^{(m)} W(t)$ satisfying $W(0)=\xi$ is the $m$ th-order approximate solution of (3) with the following error estimate.

Theorem 2. Let Assumption 1 hold. There exist positive constants $\varepsilon_{0}, C$, and $T$ such that for any $0<\varepsilon<\varepsilon_{0}$,

$$
\left\|\psi(t)-\psi_{a p p}(t)\right\| \leq C \varepsilon^{m}, \quad 0 \leq t \leq \frac{T}{\varepsilon} .
$$

Proof. Firstly, we calculate the differential of $\alpha_{t}^{(m)}(W(t))$ with respect to $t$,

$$
\begin{aligned}
\frac{d}{d t} \alpha_{t}^{(m)}(W(t))= & -i H_{0} e^{-i H_{0} t} W(t)+e^{-i H_{0} t} \frac{d}{d t} W(t) \\
& +\sum_{k=1}^{m} \varepsilon^{k} \frac{d}{d t}\left[h_{t}^{(k)} W(t)\right] .
\end{aligned}
$$

It is easy to know that

$$
\begin{aligned}
\frac{d}{d t}\left[h_{t}^{(k)} W(t)\right]= & -i H_{0} h_{t}^{(k)} W(t)+G_{k}(t) e^{-i H_{0} t} W(t) \\
& +\sum_{j=1}^{k-1} G_{k-j}(t) h_{t}^{(j)} W(t)-\sum_{j=1}^{k-1} h_{t}^{(j)} R_{k-j} W(t) \\
& -e^{-i H_{0} t} R_{k} W(t)+h_{t}^{(k)} \sum_{j=1}^{m} \varepsilon^{j} R_{j} W(t) .
\end{aligned}
$$

Since

$$
\begin{aligned}
\sum_{k=2}^{m} \varepsilon^{k} \sum_{j=1}^{k-1} G_{k-j}(t) h_{t}^{(j)} W(t) & =\sum_{k=1}^{m-1} \varepsilon^{k} G_{k}(t) \sum_{j=1}^{m-k} \varepsilon^{j} h_{t}^{(j)} W(t), \\
\sum_{k=2}^{m} \varepsilon^{k} \sum_{j=1}^{k-1} h_{t}^{(j)} R_{k-j} W(t) & =\sum_{k=1}^{m-1} \varepsilon^{k} h_{t}^{(k)} \sum_{j=1}^{m-k} \varepsilon^{j} R_{j} W(t),
\end{aligned}
$$

we have

$$
\begin{aligned}
\frac{d}{d t} \alpha_{t}^{(m)}(W(t))= & -i H_{0} \alpha_{t}^{(m)}(W(t))+\sum_{k=1}^{m} \varepsilon^{k} G_{k}(t) \alpha_{t}^{(m)}(W(t)) \\
& +\varepsilon^{m+1} S(t, \varepsilon) W(t)
\end{aligned}
$$

where

$$
S(t, \varepsilon)=\sum_{k=1}^{m} \sum_{j=1}^{k} \varepsilon^{j-1}\left(h_{t}^{(k)} R_{m-k+j}-G_{k}(t) h_{t}^{(m-k+j)}\right) .
$$

Secondly, we prove that $\alpha_{t}^{(m)}(W(t))$ is the $m$-order approximate solution satisfying (10). Define $U(t) \triangleq e^{i H_{0} t}[\psi$
$\left.(t)-\alpha_{t}^{(m)}(W(t))\right]$, we have

$$
\begin{aligned}
\frac{d U}{d t}= & \sum_{k=1}^{m} \varepsilon^{k} e^{i H_{0} t} G_{k}(t) e^{-i H_{0} t} U-\varepsilon^{m+1} e^{i H_{0} t} S(t, \varepsilon) W(t) \\
& +\varepsilon^{m+1} e^{i H_{0} t} \tilde{S}(t, \varepsilon) \psi(t),
\end{aligned}
$$

where $\tilde{S}(t, \varepsilon)=\sum_{j=1}^{\infty} \varepsilon^{j-1} G_{m+j}(t)$. The convergence of $\tilde{S}(t, \varepsilon)$ is guaranteed by the form of (3). Since $S(t, \varepsilon)$ and $\tilde{S}(t, \varepsilon)$ are quasiperiodic matrices, $e^{i H_{0} t}$ is the unitary matrix, and there exist $L>0$ and $M>0$ such that

$$
\|U(t)\| \leq \varepsilon L \int_{0}^{t}\|U(s)\| d s+\varepsilon^{m+1} M t
$$

holds. By Grönwall inequality, we have

$$
\|U(t)\| \leq \frac{M}{L} \varepsilon^{m}\left(e^{\varepsilon L t}-1\right) \leq \frac{M}{L} \varepsilon^{m}\left(e^{L T}-1\right),
$$

with $0 \leq t \leq(T / \varepsilon)$.

Finally, $\psi_{\text {app }}(t)$ is the $m$ th-order approximate solution satisfying (10). Since $e^{i H_{0} t} h_{t}^{(m)}$ is a quasiperiodic matrix, it is easy to know that

$$
\begin{aligned}
& \left\|\psi(t)-\psi_{\text {app }}(t)\right\| \leq\left\|\psi(t)-\alpha_{t}^{(m)}(W(t))\right\|+\left\|\varepsilon^{m} h_{t}^{(m)} W(t)\right\| \\
& \quad \leq C \varepsilon^{m}, \quad 0 \leq t \leq \frac{T}{\varepsilon} .
\end{aligned}
$$

Remark 3. Chiba defined $\alpha_{t}^{(m)}(W(t))$ with arbitrary integral constant as the $m$-order RG approximate solution for a more general system (see [12]). Here, we use $\psi_{\text {app }}(t)$ as the $m$-order approximate solution for the Schrödinger equation (3), which is easier to get the explicit expression for two-level systems. The selection of integral constant is suitable for numerical calculation.

\section{Two-Level Systems in and out of Resonance}

In this section, we show how to apply the RG method to twolevel systems in and out of resonance directly.

3.1. In Resonance. Let the quasi-resonant control $\mathcal{U}(t ; \varepsilon)$ $=\varepsilon u(t)$ with

$$
u(t) \triangleq v e^{i \omega t}+v^{*} e^{-i \omega t}
$$

where $\omega=\lambda_{e}-\lambda_{g}$ is the transition frequency, and $v \in \mathbb{C}$ is the complex amplitude. The two-level systems (2) becomes

$$
i \frac{d}{d t} \psi=\left(H_{0}+\varepsilon u H_{1}\right) \psi
$$

Let $\psi=\psi_{0}+\varepsilon \psi_{1}+\varepsilon^{2} \psi_{2}+\cdots$. Equating the coefficients 

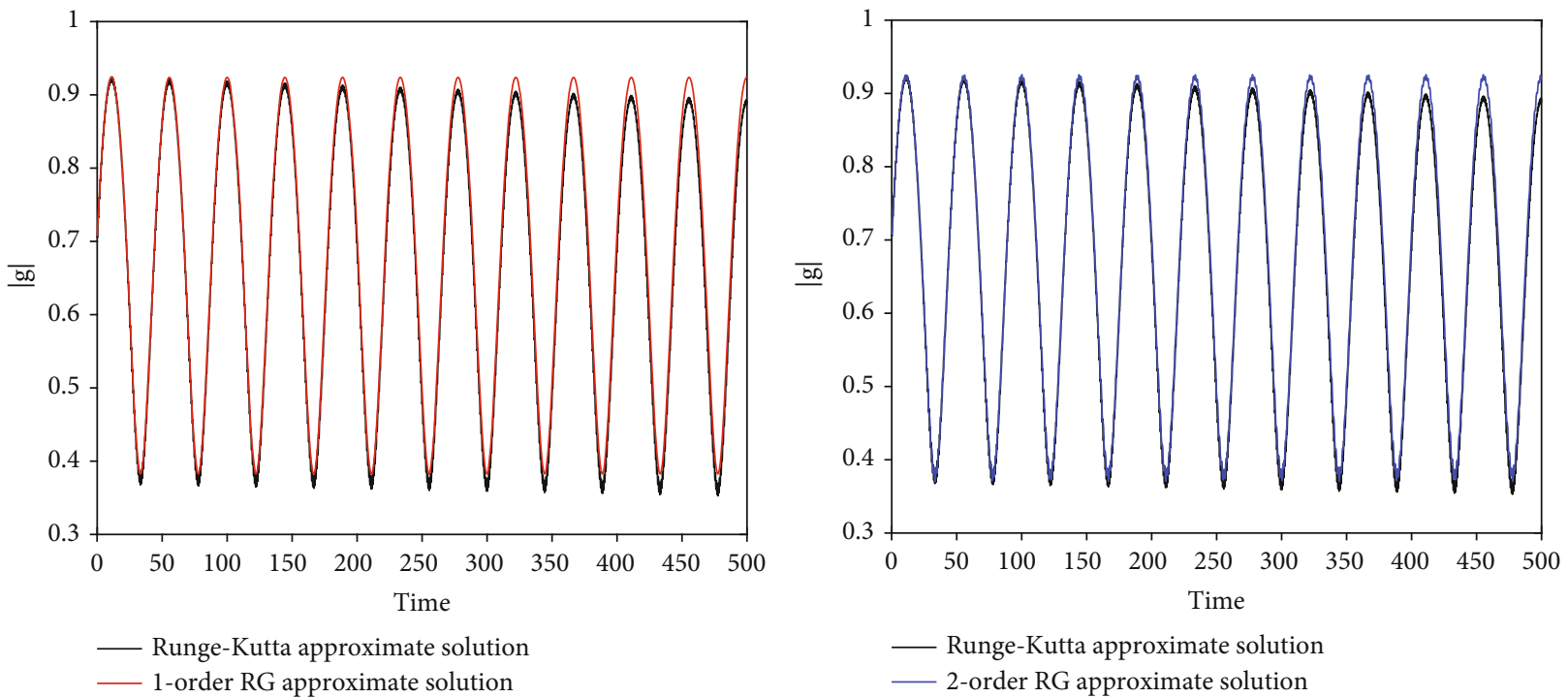

FIgURE 1: RG vs. Runge-Kutta approximate solutions of two-level systems in resonance.

of each $\varepsilon^{i}$, we have

$$
\begin{aligned}
& \varepsilon^{0}: \frac{d}{d t} \psi_{0}+i H_{0} \psi_{0}=0, \\
& \varepsilon^{k}: \frac{d}{d t} \psi_{k}+i H_{0} \psi_{k}=G_{1}(t) \psi_{k-1}, k \geq 1,
\end{aligned}
$$

where $G_{1}(t)=-i u H_{1}$.

Firstly, we calculate the first-order RG approximate solution. Since

$$
e^{i H_{0} \tau} u H_{1} e^{-i H_{0} \tau}=\left(v+v^{*} e^{-i 2 \omega \tau}\right)|g\rangle\left\langle e\left|+\left(v e^{i 2 \omega \tau}+v^{*}\right)\right| e\right\rangle\langle g|,
$$

the first-order resonant part is equal to

$$
R_{1}=\lim _{t \rightarrow \infty} \frac{1}{t} \int_{0}^{t}\left(e^{i H_{0} \tau} G_{1}(\tau) e^{-i H_{0} \tau}\right) d \tau=-i\left(v|g\rangle\left\langle e\left|+v^{*}\right| e\right\rangle\langle g|\right) .
$$

The first-order RG approximate solution is

$$
\psi_{1 \mathrm{st}}(t)=e^{-i H_{0} t} W(t), \quad 0 \leq t \leq \frac{T}{\varepsilon}
$$

where $W$ is the solution of the first-order RG equation $(d / d$ t) $W=\varepsilon R_{1} W$ with $W(0)=\xi$.

Secondly, we calculate the second-order RG approximate solution. By (7),

$$
\begin{aligned}
e^{i H_{0} t} h_{t}^{(1)} & =\int_{0}^{t}\left(e^{i H_{0} \tau} G_{1}(\tau) e^{-i H_{0} \tau}-R_{1}\right) d \tau \\
& =\frac{v^{*}}{2 \omega}\left(e^{-i 2 \omega t}-1\right)|g\rangle\left\langle e\left|-\frac{v}{2 \omega}\left(e^{i 2 \omega t}-1\right)\right| e\right\rangle\langle g| .
\end{aligned}
$$

Since

$$
\begin{aligned}
e^{i H_{0} \tau} G_{1}(\tau) h_{\tau}^{(1)}= & -i\left(\frac{|v|^{2}}{2 \omega}\left(1-e^{i 2 \omega \tau}\right)+\frac{v^{* 2}}{2 \omega}\left(e^{-i 2 \omega \tau}-1\right)\right)|e\rangle \\
& \cdot\left\langle e\left|+i\left(\frac{v^{2}}{2 \omega}\left(e^{i 2 \omega \tau}-1\right)+\frac{|v|^{2}}{2 \omega}\left(1-e^{-i 2 \omega \tau}\right)\right)\right| g\right\rangle\langle g|, \\
e^{i H_{0} \tau} h_{\tau}^{(1)} R_{1}= & -i\left(\frac{v^{* 2}}{2 \omega}\left(e^{-i 2 \omega t}-1\right)|g\rangle\left\langle g\left|-\frac{v^{2}}{2 \omega}\left(e^{i 2 \omega t}-1\right)\right| e\right\rangle\langle e|\right) .
\end{aligned}
$$

Now, the second-order resonant part is equal to by (6),

$$
\begin{aligned}
R_{2} & =\lim _{t \rightarrow \infty} \frac{1}{t} \int_{0}^{t}\left(e^{i H_{0} \tau} G_{2} \tau e^{-i H_{0} \tau}+e^{i H_{0} \tau} G_{1}(\tau) h_{\tau}^{(1)}-e^{i H_{0} \tau} h_{\tau}^{(1)} R_{1}\right) d \tau \\
& =-i \frac{|v|^{2}-v^{2}-v^{* 2}}{2 \omega}(|e\rangle\langle e|-| g\rangle\langle g|) .
\end{aligned}
$$

Thus, the second-order RG approximate solution is

$$
\psi_{2 \mathrm{nd}}(t)=e^{-i H_{0} t} W(t)+\varepsilon h_{t}^{(1)} W(t), \quad 0 \leq t \leq \frac{T}{\varepsilon},
$$

where $W$ is the solution of the second-order RG equation $(d / d t) W=\varepsilon R_{1} W+\varepsilon^{2} R_{2} W$ with $W(0)=\xi$.

Example 4. Consider the two-level system (21) with initial value $\xi=(\sqrt{2} / 2, \sqrt{2} / 2)$. Let $\lambda_{e}=5, \lambda_{g}=1, v=1+i$, and $\varepsilon=$ 0.05 . The simulations of Figure 1 illustrate that first-order RG approximate solution is as good as the second-order RG approximate solution.

3.2. Out of Resonance. We take the quasi-resonant control $\mathcal{U}(t ; \varepsilon)=\varepsilon u(t)$ with

$$
u(t) \triangleq v e^{i(\omega-\Delta) t}+v^{*} e^{-i(\omega-\Delta) t},
$$


where $\omega=\lambda_{e}-\lambda_{g}$ is the transition frequency, $\Delta$ is the detuning frequency, and $v \in \mathbb{C}$ is the complex amplitude. The two-level system (2) becomes

$$
i \frac{d}{d t} \psi=\left(H_{0}+\varepsilon u H_{1}\right) \psi
$$

Let $\psi=\psi_{0}+\varepsilon \psi_{1}+\varepsilon^{2} \psi_{2}+\cdots$. Equating the coefficients of each $\varepsilon^{i}$, we have

$$
\begin{aligned}
& \varepsilon^{0}: \frac{d}{d t} \psi_{0}+i H_{0} \psi_{0}=0, \\
& \varepsilon^{k}: \frac{d}{d t} \psi_{k}+i H_{0} \psi_{k}=G_{1}(t) \psi_{k-1}, k \geq 1,
\end{aligned}
$$

where $G_{1}(t)=-i u H_{1}$.

Firstly, we calculate the first-order RG approximate solution. Since

$$
\begin{aligned}
e^{i H_{0} \tau} u H_{1} e^{-i H_{0} \tau}= & \left(v e^{-i \Delta \tau}+v^{*} e^{-i(2 \omega-\Delta) \tau}\right)|g\rangle \\
& \cdot\left\langle e\left|+\left(v e^{i(2 \omega-\Delta) \tau}+v^{*} e^{i \Delta \tau}\right)\right| e\right\rangle\langle g|,
\end{aligned}
$$

the first-order resonant part is equal to

$$
R_{1}=\lim _{t \rightarrow \infty} \frac{1}{t} \int_{0}^{t}\left(e^{i H_{0} \tau} G_{1}(\tau) e^{-i H_{0} \tau}\right) d \tau=0 .
$$

The first-order RG approximate solution is

$$
\psi_{1 \mathrm{st}}(t)=e^{-i H_{0} t} W(t), \quad 0 \leq t \leq \frac{T}{\varepsilon}
$$

where $W$ is the solution of the first-order RG equation $(d / d$ t) $W=\varepsilon R_{1} W$ with $W(0)=\xi$.

Secondly, we calculate the second-order RG approximate solution. By (7),

$$
\begin{aligned}
e^{i H_{0} t} h_{t}^{(1)}= & \int_{0}^{t}\left(e^{i H_{0} \tau} G_{1}(\tau) e^{-i H_{0} \tau}-R_{1}\right) d \tau \\
= & \left(\frac{v}{\Delta}\left(e^{-i \Delta t}-1\right)+\frac{v^{*}}{2 \omega-\Delta}\left(e^{-i(2 \omega-\Delta) t}-1\right)\right)|g\rangle \\
& \cdot\left\langle e\left|-\left(\frac{v}{2 \omega-\Delta}\left(e^{i(2 \omega-\Delta) t}-1\right)+\frac{v^{*}}{\Delta}\left(e^{i \Delta t}-1\right)\right)\right| e\right\rangle\langle g| .
\end{aligned}
$$

Since

$$
\begin{aligned}
e^{i H_{0} \tau} G_{1}(\tau) h_{\tau}^{(1)}= & -i\left(\frac{v^{2}}{\Delta}\left(e^{i 2(\omega-\Delta) \tau}-e^{i(2 \omega-\Delta) \tau}\right)+\frac{|v|^{2}}{\Delta}\left(1-e^{i \Delta \tau}\right)\right. \\
& +\frac{|v|^{2}}{2 \omega-\Delta}\left(1-e^{i(2 \omega-\Delta) \tau}\right) \\
& \left.+\frac{v^{* 2}}{2 \omega-\Delta}\left(e^{-i(2 \omega-2 \Delta) \tau}-e^{i \Delta \tau}\right)\right)|e\rangle \\
& \cdot\langle e|+i\left(\frac{v^{2}}{2 \omega-\Delta}\left(e^{i(2 \omega-2 \Delta) \tau}-e^{-i \Delta \tau}\right)\right. \\
& +\frac{|v|^{2}}{2 \omega-\Delta}\left(1-e^{-i(2 \omega-\Delta) \tau}\right)+\frac{|v|^{2}}{\Delta}\left(1-e^{-i \Delta \tau}\right) \\
& \left.+\frac{v^{* 2}}{\Delta}\left(e^{-i(2 \omega-2 \Delta) \tau}-e^{-i(2 \omega-\Delta) \tau}\right)\right)|g\rangle\langle g|,
\end{aligned}
$$

the second-order resonant part is equal to

$$
\begin{aligned}
R_{2} & =\lim _{s \rightarrow \infty} \frac{1}{s} \int_{0}^{s}\left(e^{i H_{0} \tau} G_{2}(\tau) e^{-i H_{0} \tau}+e^{i H_{0} \tau} G_{1}(\tau) h_{\tau}^{(1)}-e^{i H_{0} \tau} h_{\tau}^{(1)} R_{1}\right) d \tau \\
& =-i\left(\left(\frac{|v|^{2}}{\Delta}+\frac{|v|^{2}}{2 \omega-\Delta}\right)(|e\rangle\langle e|-| g\rangle\langle g|)\right) .
\end{aligned}
$$

Thus, the second-order RG approximate solution is

$$
\psi_{2 \mathrm{nd}}(t)=e^{-i H_{0} t} W(t)+\varepsilon h_{t}^{(1)} W(t), \quad 0 \leq t \leq \frac{T}{\varepsilon},
$$

where $W$ is the solution of the second-order RG equation $(d / d t) W=\varepsilon R_{1} W+\varepsilon^{2} R_{2} W$ with $W(0)=\xi$.

Example 5. Consider the two-level system (31) with initial value $\xi=(\sqrt{2} / 2, \sqrt{2} / 2)$. Let $\lambda_{e}=5, \lambda_{g}=1, v=1+i, \varepsilon=0.05$, and $\Delta=1$. The simulations of Figure 2 illustrate that the first-order approximate solution is not a reliable one in the two-level system out of resonance, while the second-order approximate solution is a better one.

\section{Near Resonance Problems with Two Scales}

In this section, we will discuss how to apply RG method reasonably to near resonance problem with two scales. Take the quasi-resonant control $\mathcal{U}(t ; \varepsilon)=\varepsilon \mathcal{\varepsilon}(t ; \varepsilon)$ with

$$
u(t ; \varepsilon) \triangleq v e^{i(\omega-\varepsilon \delta) t}+v^{*} e^{-i(\omega-\varepsilon \delta) t}
$$

where $\omega=\lambda_{e}-\lambda_{g}$ is the transition frequency, $\delta$ is the detuning frequency, and $v \in \mathbb{C}$ is the complex amplitude. The twolevel system (2) becomes

$$
i \frac{d}{d t} \psi=\left(H_{0}+\varepsilon u H_{1}\right) \psi
$$

4.1. Invalid Expansion. $|g\rangle\left\langle g\left|+\lambda_{g}\right| g\right\rangle\langle g|$ An intuitive way to 


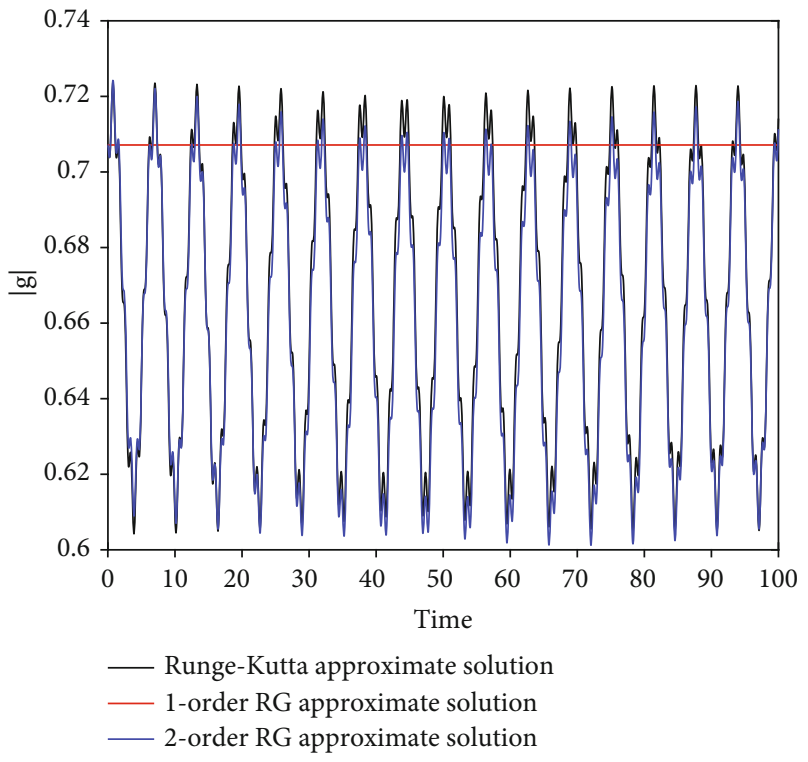

FIGURE 2: RG vs. Runge-Kutta approximate solutions of two-level systems out of resonance.

deal with such problems is to divide $H_{0}$ into two parts:

$$
H_{0}=\left(\lambda_{e}|e\rangle\left\langle e\left|\lambda_{g}\right| g\right\rangle\langle g|+\varepsilon \delta| g\rangle\langle g|\right)+(-\varepsilon \delta|g\rangle\langle g|) .
$$

Let $\tilde{H}_{0}=\lambda_{e}|e\rangle\left\langle e\left|+\lambda_{g}\right| g\right\rangle\langle g|+\varepsilon \delta| g\rangle\langle g|, \quad \tilde{\omega}=\omega-\varepsilon \delta$, and $\phi=\phi_{0}+\varepsilon \phi_{1}+\varepsilon^{2} \phi_{2}+\cdots$. Formally, we have the following expansion

$$
\begin{aligned}
& \varepsilon^{0}: \frac{d}{d t} \phi_{0}+i \tilde{H}_{0} \phi_{0}=0, \\
& \varepsilon^{k}: \frac{d}{d t} \phi_{k}+i \tilde{H}_{0} \phi_{k}=G_{1}(t) \phi_{k-1}, k \geq 1,
\end{aligned}
$$

with $G_{1}(t)=-i\left(-\delta|g\rangle\langle g|+u H_{1}\right)$, where $u=v e^{i \tilde{\omega} t}+v^{*} e^{-i \tilde{\omega} t}$. Because there are actually small parameters in $\tilde{H}_{0}$ and $u$, we call the above expression invalid expansion.

If we calculate the first-order RG approximate solution formally, we have the first-order resonant part $|g\rangle\left\langle e\left|+v^{*}\right|\right.$ $e\rangle\langle g|$

$$
\begin{aligned}
R_{1} & =\lim _{t \rightarrow \infty} \frac{1}{s} \int_{0}^{t}\left(e^{i \tilde{H}_{0} \tau} G_{1}(\tau) e^{-i \tilde{H}_{0} \tau}\right) d \tau \\
& =-i\left(-\delta|g\rangle\langle g|+v| g\rangle\left\langle e\left|+v^{*}\right| e\right\rangle\langle g|\right),
\end{aligned}
$$

which is exactly the result obtained by RWA (see [19]). Here, the first-order RG approximate solution can be expressed as follows.

$$
\phi_{1 s t}=e^{-i \tilde{H}_{0} t} W(t), \quad 0 \leq t \leq \frac{T}{\varepsilon},
$$

where $W$ is the solution of the first-order RG equation $(d / d t) W=\varepsilon R_{1} W$ with $W(0)=\xi$.
4.2. Valid Expansion. Although the so-called invalid expansion is sometimes a good approximation, shall we construct a valid expansion and find a more reliable long-time higher-order approximate solution? Here, we introduce a quasi-resonant time transformation technique $s \triangleq(1-\varepsilon \kappa) t$ with $\kappa=\delta / \omega$. Now, the system (41) is equal to

$$
(1-\varepsilon \kappa) \frac{d}{d s} \psi=-i\left(H_{0}+\varepsilon u H_{1}\right) \psi
$$

where $u$ is the resonant control with respect to $s$ :

$$
u(s)=v e^{i \omega s}+v^{*} e^{-i \omega s} .
$$

Let $\psi=\psi_{0}+\varepsilon \psi_{1}+\varepsilon^{2} \psi_{2}+\cdots$. Equating the coefficients of each $\varepsilon^{i}$, we have

$$
\begin{aligned}
& \varepsilon^{0}: \frac{d}{d s} \psi_{0}+i H_{0} \psi_{0}=0, \\
& \varepsilon^{1}: \frac{d}{d s} \psi_{1}+i H_{0} \psi_{1}=G_{1}(s) \psi_{0}, \\
& \varepsilon^{2}: \frac{d}{d s} \psi_{2}+i H_{0} \psi_{2}=G_{1}(s) \psi_{1}+G_{2}(s) \psi_{0},
\end{aligned}
$$

where $G_{k}(s)=-i \kappa^{k-1}\left(\kappa H_{0}+u H_{1}\right)$.

Firstly, we calculate the first-order RG approximate solution.

$$
R_{1}=\lim _{s \rightarrow \infty} \frac{1}{s} \int_{0}^{s}\left(e^{i H_{0} \tau} G_{1}(\tau) e^{-i H_{0} \tau}\right) d \tau=-i\left(\kappa H_{0}+H_{2}\right),
$$

where $\mathrm{H}_{2} \triangleq v|g\rangle\left\langle e\left|+v^{*}\right| e\right\rangle\langle g|$. Thus, the first-order RG equation and first-order RG approximate solution are

$$
\frac{d}{d s} W=\varepsilon R_{1} W \text { and } \psi_{1 s t}=e^{-i H_{0} s} W(s), \quad 0 \leq s \leq \frac{T}{\varepsilon},
$$

respectively, where $w(0)=\xi$, and $s=(1-\varepsilon \kappa) t$.

Secondly, we calculate the second-order RG approximate solution. By (7),

$$
\begin{aligned}
e^{i H_{0} s} h_{s}^{(1)} & =\int_{0}^{s}\left(e^{i H_{0} \tau} G_{1}(\tau) e^{-i H_{0} \tau}-R_{1}\right) d \tau \\
& =\frac{v^{*}}{2 \omega}\left(e^{-i 2 \omega s}-1\right)|g\rangle\left\langle e\left|\frac{v}{2 \omega}\left(e^{i 2 \omega s}-1\right)\right| e\right\rangle\langle g| . .
\end{aligned}
$$


Since

$$
\begin{aligned}
e^{i H_{0} \tau} G_{1}(\tau) h_{\tau}^{(1)}= & -i\left(\frac{|v|^{2}}{2 \omega}\left(1-e^{i 2 \omega \tau}\right)|e\rangle\left\langle e\left|-\frac{|v|^{2}}{2 \omega}\left(1-e^{-i 2 \omega \tau}\right)\right| g\right\rangle\langle g|\right. \\
& +\frac{v^{* 2}}{2 \omega}\left(e^{-i 2 \omega \tau}-1\right)|e\rangle\left\langle e\left|-\frac{v^{2}}{2 \omega}\left(e^{i 2 \omega \tau}-1\right)\right| g\right\rangle\langle g| \\
& \left.+\kappa \lambda_{g} \frac{v^{*}}{2 \omega}\left(e^{-i 2 \omega \tau}-1\right)|g\rangle\left\langle e\left|-\kappa \lambda_{e} \frac{v}{2 \omega}\left(e^{i 2 \omega \tau}-1\right)\right| e\right\rangle\langle g|\right), \\
e^{i H_{0} \tau} h_{\tau}^{(1)} R_{1}= & -i\left(\kappa \lambda_{e} \frac{v^{*}}{2 \omega}\left(e^{-i 2 \omega \tau}-1\right)|g\rangle\left\langle e\left|-\kappa \lambda_{g} \frac{v}{2 \omega}\left(e^{i 2 \omega \tau}-1\right)\right| e\right\rangle\langle g|\right. \\
& \left.+\frac{v^{* 2}}{2 \omega}\left(e^{-i 2 \omega \tau}-1\right)|g\rangle\left\langle g\left|-\frac{v^{2}}{2 \omega}\left(e^{i 2 \omega \tau}-1\right)\right| e\right\rangle\langle e|\right),
\end{aligned}
$$

the second-order resonant part is equal to

$$
\begin{aligned}
R_{2}= & \lim _{s \rightarrow \infty} \frac{1}{s} \int_{0}^{s}\left(e^{i H_{0} \tau} G_{2}(\tau) e^{-i H_{0} \tau}+e^{i H_{0} \tau} G_{1}(\tau) h_{\tau}^{(1)}\right. \\
& \left.-e^{i H_{0} \tau} h_{\tau}^{(1)} R_{1}\right) d \tau=-i\left(\kappa^{2} H_{0}+\kappa H_{2}\right. \\
& +\kappa \frac{v^{*}}{2}|g\rangle\left\langle e\left|+\kappa \frac{v}{2}\right| e\right\rangle\langle g| \\
& \left.+\frac{|v|^{2}-v^{2}-v^{* 2}}{2 \omega}(|e\rangle\langle e|-| g\rangle\langle g|)\right) .
\end{aligned}
$$

Thus, the second-order RG equation and the secondorder RG approximate solution are

$$
\begin{aligned}
\frac{d}{d s} W & =\varepsilon R_{1} W+\varepsilon^{2} R_{2} W \text { and } \psi_{2 \text { nd }} \\
& =e^{-i H_{0} s} W(s)+\varepsilon h_{s}^{(1)} W(s), \quad 0 \leq s \leq \frac{T}{\varepsilon},
\end{aligned}
$$

respectively, where $W(0)=\xi$, and $s=(1-\varepsilon \kappa) t$.

Example 6. Consider the two-level system (41) with initial value $\xi=(\sqrt{2} / 2, \sqrt{2} / 2)$. Let $\lambda_{e}=5, \lambda_{g}=1, v=1+i, \varepsilon=0.05$, and $\delta=1$. The simulations of Figure 3 illustrate that the first-order approximate solution is not good enough in twolevel systems near resonance. But the second-order approximate solution is a more reliable one.

Example 7. Consider a spin-half system

$$
\frac{d}{d t} \psi=-i\left(\frac{\omega_{e g}}{2} \sigma_{z}+\frac{v}{2}\left(e^{i\left(\omega_{e g}-\Delta\right) t}+e^{-i\left(\omega_{e g}-\Delta\right) t}\right) \sigma_{x}\right) \psi
$$

where $v \sim O(\varepsilon)$ and $\Delta \sim O(\varepsilon)$ are real numbers, $\sigma_{z}=|e\rangle\langle e|-|$ $g\rangle\left\langle g\left|, \sigma_{x}=\right| g\right\rangle\langle e|+| e\rangle\langle g|$, and $\omega_{e g}$ is the energy difference between $|e\rangle$ and $|g\rangle$. Let $\lambda_{e}=\omega_{e g} / 2$, and $\lambda_{g}=-\omega_{e g} / 2$, we have

$$
H_{0}=\frac{\omega_{e g}}{2}(|e\rangle\langle e|-| g\rangle\langle g|)=\frac{\omega_{e g}}{2} \sigma_{z} .
$$
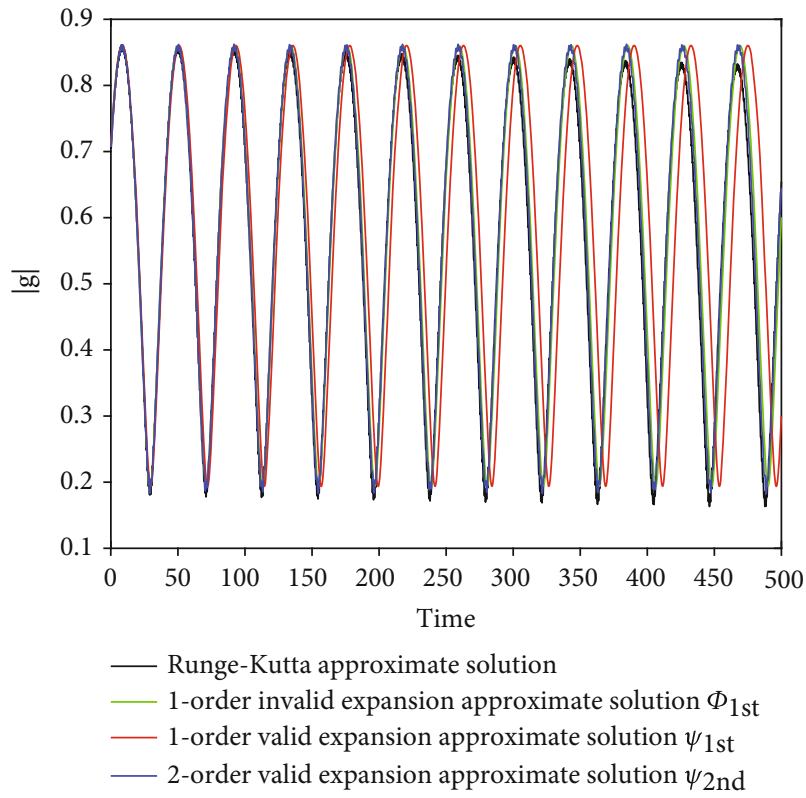

FIGURE 3: Valid and invalid RG approximate solutions of two-level systems near resonance.

The first-order RG equation is equal to

$$
\frac{\mathrm{d} W}{\mathrm{~d} s}=-i\left(\frac{\Delta}{2} \sigma_{z}+\frac{v}{2} \sigma_{x}\right) W,
$$

where $s=\left(1-\left(\Delta / \omega_{e g}\right)\right) t, v$, and $\Delta$ are Rabi frequency and detuning, respectively. The first-order generalized Rabi frequency is

$$
\Omega_{1 \mathrm{st}}=\sqrt{v^{2}+\Delta^{2}} .
$$

Furthermore, we have the second-order RG equation

$$
\frac{\mathrm{d} W}{\mathrm{~d} s}=-i\left[\left(\frac{\Delta}{2}+\frac{\Delta^{2}}{2 \omega_{e g}}-\frac{v^{2}}{8 \omega_{e g}}\right) \sigma_{z}+\left(\frac{v}{2}+\frac{3 \Delta v}{4 \omega_{e g}}\right) \sigma_{x}\right] W .
$$

Remark 8. Our method can be applied to the spin-half system directly. In this article, we only take near resonance problems with two scales as an example.

\section{Near Resonance Problems with Three Scales}

Physicists like to use " $\gg$ " to indicate different time scales. If there are more than one " $\gg$ " relationships in near resonance problems, how can we analyze the long-time dynamic behavior reasonably? In this section, we shall apply our method to two-level systems in high-order near resonant case and highorder weak driving case. 
5.1. High-Order Near Resonant Case. Let $\mathscr{U}(t ; \varepsilon)=\varepsilon u(t ; \varepsilon)$ with

$$
u(t ; \varepsilon) \triangleq v e^{i\left(\omega-\varepsilon^{2} \delta\right) t}+v^{*} e^{-i\left(\omega-\varepsilon^{2} \delta\right) t} .
$$

Introduce a quasi-resonant time transformation $s \triangleq(1$ $\left.-\varepsilon^{2} \kappa\right) t$ with $\kappa=\delta / \omega$. The two-level system (2) becomes

$$
\left(1-\varepsilon^{2} \kappa\right) \frac{d}{d s} \psi=-i\left(H_{0}+\varepsilon u H_{1}\right) \psi
$$

where $u$ is the resonant control with respect to $s$ :

$$
u(s)=v e^{i \omega s}+v^{*} e^{-i \omega s} .
$$

Let $\psi=\psi_{0}+\varepsilon \psi_{1}+\varepsilon^{2} \psi_{2}+\cdots$. Equating the coefficients of each $\varepsilon^{i}$, we have

$$
\begin{aligned}
& \varepsilon^{0}: \frac{d}{d s} \psi_{0}+i H_{0} \psi_{0}=0, \\
& \varepsilon^{1}: \frac{d}{d s} \psi_{1}+i H_{0} \psi_{1}=G_{1}(s) \psi_{0}, \\
& \varepsilon^{2}: \frac{d}{d s} \psi_{2}+i H_{0} \psi_{2}=G_{1}(s) \psi_{1}+G_{2}(s) \psi_{0},
\end{aligned}
$$

where $G_{1}(s)=-i u H_{1}$, and $G_{2}(s)=-i \kappa H_{0}$.

Firstly, we calculate the first-order RG approximate solution.

$$
R_{1}=\lim _{s \rightarrow \infty} \frac{1}{s} \int_{0}^{s}\left(e^{i H_{0} \tau} G_{1}(\tau) e^{-i H_{0} \tau}\right) d \tau=-i H_{2} .
$$

Thus, the first-order RG equation and the first-order RG approximate solution are

$$
\frac{d}{d s} W=\varepsilon R_{1} W \text { and } \psi_{1 \mathrm{st}}=e^{-i H_{0} s} W(s), \quad 0 \leq s \leq \frac{T}{\varepsilon},
$$

respectively, where $W(0)=\xi$, and $s=\left(1-\varepsilon^{2} \kappa\right) t$.

Secondly, we calculate the second-order RG approximate solution. By (7)

$$
\begin{aligned}
e^{i H_{0} s} h_{s}^{(1)} & =\int_{0}^{s}\left(e^{i H_{0} \tau} G_{1}(\tau) e^{-i H_{0} \tau}-R_{1}\right) d \tau \\
& =\frac{v^{*}}{2 \omega}\left(e^{-i 2 \omega s}-1\right)|g\rangle\left\langle e\left|-\frac{v}{2 \omega}\left(e^{i 2 \omega s}-1\right)\right| e\right\rangle\langle g| . .
\end{aligned}
$$

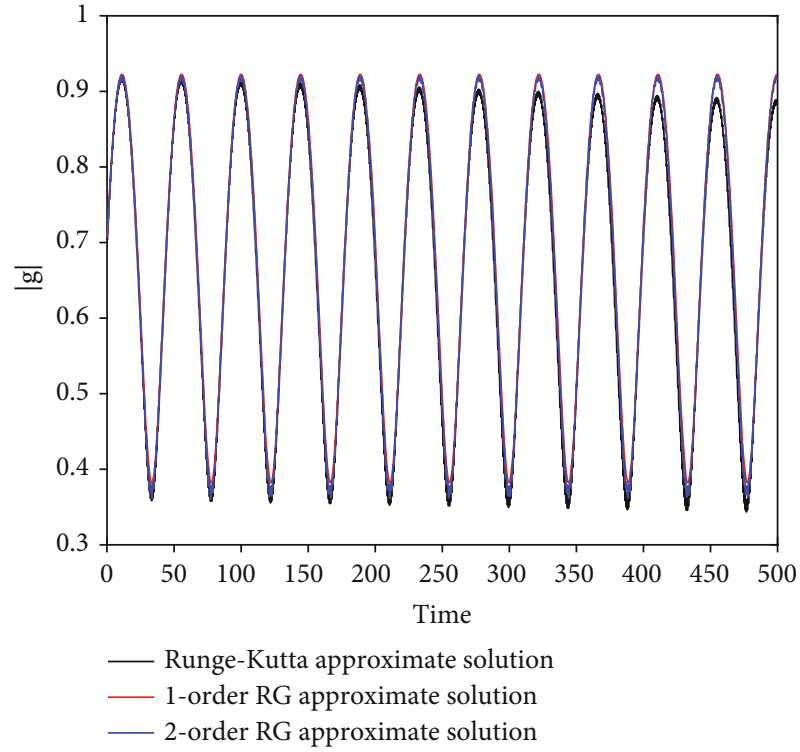

Figure 4: RG vs. Runge-Kutta approximate solutions of two-level systems in high-order quasiresonant case.

The second-order resonant part is equal to

$$
\begin{aligned}
R_{2}= & \lim _{s \rightarrow \infty} \frac{1}{s} \int_{0}^{s}\left(e^{i H_{0} \tau} G_{2}(\tau) e^{-i H_{0} \tau}+e^{i H_{0} \tau} G_{1}(\tau) h_{\tau}^{(1)}\right. \\
& \left.-e^{i H_{0} \tau} h_{\tau}^{(1)} R_{1}\right) d \tau=-i\left(\kappa H_{0}\right. \\
& \left.+\frac{|v|^{2}-v^{2}-v^{* 2}}{2 \omega}(|e\rangle\langle e|-| g\rangle\langle g|)\right) .
\end{aligned}
$$

Thus, the second-order RG equation and the secondorder RG approximate solution are

$$
\begin{aligned}
\frac{d}{d s} W & =\varepsilon R_{1} W+\varepsilon^{2} R_{2} W \text { and } \psi_{2 \text { nd }} \\
& =e^{-i H_{0} s} W(s)+\varepsilon h_{s}^{(1)} W(s), \quad 0 \leq s \leq \frac{T}{\varepsilon},
\end{aligned}
$$

respectively, where $W(0)=\xi$, and $s=\left(1-\varepsilon^{2} \kappa\right) t$.

Example 9. Consider the two-level system in high-order near resonance case with initial value $\xi=(\sqrt{2} / 2, \sqrt{2} / 2)$. Let $\lambda_{e}=5$, $\lambda_{g}=1, v=1+i, \varepsilon=0.05$, and $\delta=1$. The simulations of Figure 4 illustrate that the first-order approximate solution is reliable in two-level systems with high-order near resonant control.

5.2. High-Order Weak Driving Case. Let $\mathscr{U}(t ; \varepsilon)=\varepsilon^{2} u(t ; \varepsilon)$ with

$$
u(t ; \varepsilon) \triangleq v e^{i(\omega-\varepsilon \delta) t}+v^{*} e^{-i(\omega-\varepsilon \delta) t} .
$$

Introduce a quasi-resonant time transformation $s \triangleq(1$ 
$-\varepsilon \kappa) t$ with $\kappa=\delta / \omega$. The two-level system (2) becomes

$$
(1-\varepsilon \kappa) \frac{d}{d s} \psi=-i\left(H_{0}+\varepsilon^{2} u H_{1}\right) \psi
$$

Now $u$ is the resonant control with respect to $s$ :

$$
u(s)=v e^{i \omega s}+v^{*} e^{-i \omega s} .
$$

Let $\psi=\psi_{0}+\varepsilon \psi_{1}+\varepsilon^{2} \psi_{2}+\cdots$. Equating the coefficients of each $\varepsilon^{i}$, we have

$$
\begin{aligned}
& \varepsilon^{0}: \frac{d}{d s} \psi_{0}+i H_{0} \psi_{0}=0, \\
& \varepsilon^{1}: \frac{d}{d s} \psi_{1}+i H_{0} \psi_{1}=G_{1}(s) \psi_{0}, \\
& \varepsilon^{2}: \frac{d}{d s} \psi_{2}+i H_{0} \psi_{2}=G_{1}(s) \psi_{1}+G_{2}(s) \psi_{0},
\end{aligned}
$$

where $G_{1}(s)=-i \kappa H_{0}$, and $G_{2}(s)=-i\left(\kappa^{2} H_{0}+u H_{1}\right)$.

Firstly, we calculate the first-order RG approximate solution.

$$
R_{1}=\lim _{s \rightarrow \infty} \frac{1}{s} \int_{0}^{s}\left(e^{i H_{0} \tau} G_{1}(\tau) e^{-i H_{0} \tau}\right) d \tau=-i \kappa H_{0} .
$$

Thus, the first-order RG equation and the first-order RG approximate solution are

$$
\frac{d}{d s} W=\varepsilon R_{1} W \text { and } \psi_{1 s t}=e^{-i H_{0} s} W(s), \quad 0 \leq s \leq \frac{T}{\varepsilon},
$$

respectively, where $W(0)=\xi$, and $s=(1-\varepsilon \kappa) t$.

Secondly, we calculate the second-order RG approximate solution. By (7),

$$
e^{i H_{0} s} h_{s}^{(1)}=\int_{0}^{s}\left(e^{i H_{0} \tau} G_{1}(\tau) e^{-i H_{0} \tau}-R_{1}\right) d \tau=0,
$$

the second-order resonant part is equal to

$$
\begin{aligned}
R_{2}= & \lim _{s \rightarrow \infty} \frac{1}{s} \int_{0}^{s}\left(e^{i H_{0} \tau} G_{2}(\tau) e^{-i H_{0} \tau}+e^{i H_{0} \tau} G_{1}(\tau) h_{\tau}^{(1)}\right. \\
& \left.-e^{i H_{0} \tau} h_{\tau}^{(1)} R_{1}\right) d \tau=-i\left(\kappa^{2} H_{0}+H_{2}\right) .
\end{aligned}
$$

Thus, the second-order RG equation and the secondorder RG approximate solution are

$$
\begin{aligned}
\frac{d}{d s} W & =\varepsilon R_{1} W+\varepsilon^{2} R_{2} W \text { and } \psi_{2 \text { nd }} \\
& =e^{-i H_{0} s} W(s)+\varepsilon h_{s}^{(1)} W(s), \quad 0 \leq s \leq \frac{T}{\varepsilon}
\end{aligned}
$$

respectively, where $W(0)=\xi$, and $s=(1-\varepsilon \kappa) t$.

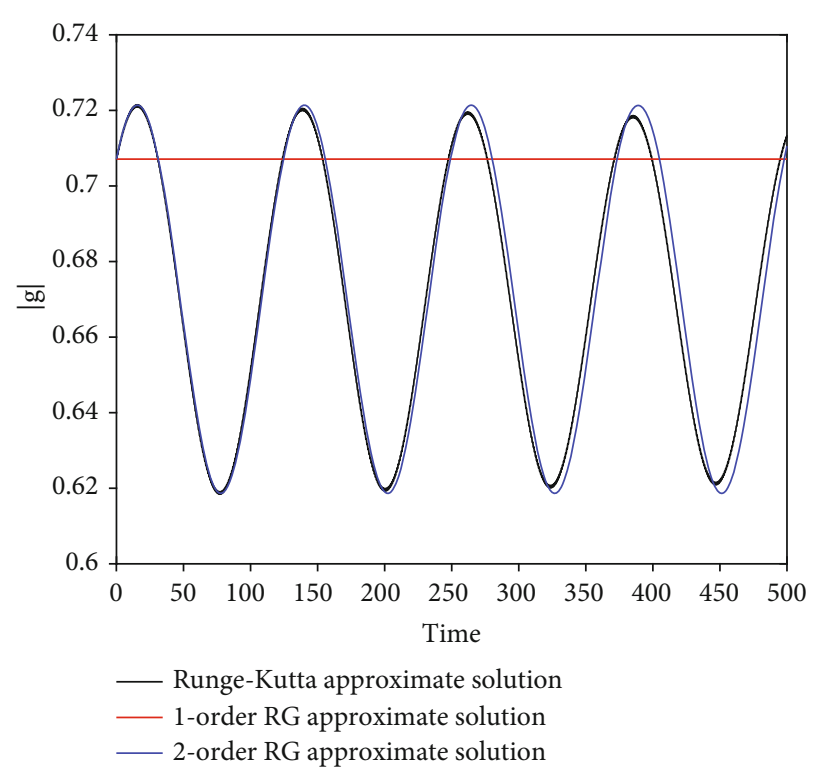

Figure 5: RG vs. Runge-Kutta approximate solutions of two-level systems in high-order weak driving case.

Example 10. Consider the two-level system in high-order weak driving case with initial value $\xi=(\sqrt{2} / 2, \sqrt{2} / 2)$. Let $\lambda_{e}$ $=5, \lambda_{g}=1, v=1+i, \varepsilon=0.05$, and $\delta=1$. The simulations of Figure 5 illustrate that the first-order approximate solution is not reliable in two-level systems with the high-order weak driving control. But the second-order approximate solution is a more accurate one.

\section{Data Availability}

The data used to support the findings of this study are included within the article.

\section{Conflicts of Interest}

The authors declare that they have no conflicts of interest.

\section{Acknowledgments}

The first author was supported by NSFC (grant numbers $11901052,11626043)$ and the Scientific and Technological Project of Jilin Province Education Department in Thirteenth Five-Year (grant number JJKH20170535KJ). The second author was supported by NSFC (grant numbers 11871140, 11401089).

\section{References}

[1] H. Serge and R. Jean-Michel, Exploring the quantum: atoms, cavities, and photons, Oxford U. Press, New York, 2006.

[2] K. Fujii, "Introduction to the rotating wave approximation (RWA): two coherent oscillations," Journal of Modern Physics, vol. 8, no. 12, pp. 2042-2058, 2017.

[3] A. B. Klimov, I. Sainz, and S. M. Chumakov, "Resonance expansion versus the rotating-wave approximation," Physical Review A, vol. 68, no. 6, article 063811, 2003. 
[4] K. R. Rao and D. Suter, "Nonlinear dynamics of a two-level system of a single spin driven beyond the rotating-wave approximation," Physical Review A, vol. 95, no. 5, article 053804, 2017.

[5] T. Werlang, A. V. Dodonov, E. I. Duzzioni, and C. J. Villas-Bôas, "Rabi model beyond the rotating-wave approximation: generation of photons from vacuum through decoherence," Physical Review A, vol. 78, no. 5, article 053805, 2008.

[6] M. Mazyar and R. Pierre, Dynamics and control of open quantum systems, 2015.

[7] L.-Y. Chen, N. Goldenfeld, and Y. Oono, "Renormalization group theory for global asymptotic analysis," Physical Review Letters, vol. 73, no. 10, pp. 1311-1315, 1994.

[8] L.-Y. Chen, N. Goldenfeld, and Y. Oono, "Renormalization group and singular perturbations: multiple scales, boundary layers, and reductive perturbation theory," Physical Review E, vol. 54, no. 1, pp. 376-394, 1996.

[9] M. Ziane, "On a certain renormalization group method," Journal of Mathematical Physics, vol. 41, no. 5, pp. 3290-3299, 2000.

[10] D. V. RL, A. Harkin, M. Holzer, K. Josić, and T. J. Kaper, "Analysis of a renormalization group method and normal form theory for perturbed ordinary differential equations," Physica D, vol. 237, no. 8, pp. 1029-1052, 2008.

[11] H. Chiba, " $\$ \mathrm{C}^{\wedge} 1 \$$ approximation of vector fields based on the renormalization group method," SIAM Journal on Applied Dynamical Systems, vol. 7, no. 3, pp. 895-932, 2008.

[12] H. Chiba, "Extension and unification of singular perturbation methods for ODEs based on the renormalization group method," SIAM Journal on Applied Dynamical Systems, vol. 8, no. 3, pp. 1066-1115, 2009.

[13] H. Chiba, "Simplified renormalization group method for ordinary differential equations," Journal of Differential Equations, vol. 246, no. 5, pp. 1991-2019, 2009.

[14] M. Frasca, "Theory of quantum resonance: a renormalizationgroup approach," Physical Review A, vol. 58, no. 1, pp. 771774, 1998.

[15] W. Li and S. Shi, "Singular perturbed renormalization group theory and its application to highly oscillatory problems," Discrete \& Continuous Dynamical Systems-B, vol. 23, no. 4, pp. 1819-1833, 2018.

[16] I. Moise and M. Ziane, "Renormalization group method applications to partial differential equations," Journal of Dynamics and Differential Equations, vol. 13, no. 2, pp. 275-321, 2001.

[17] R. E. O'Malley Jr. and E. Kirkinis, "Variation of parameters and the renormalization group method," Studies in Applied Mathematics, vol. 134, no. 2, pp. 215-232, 2015.

[18] M. Petcu, R. Temam, and D. Wirosoetisno, "Averaging method applied to the three-dimensional primitive equations," Discrete and Continuous Dynamical Systems, vol. 36, no. 10, pp. 5681-5707, 2016.

[19] M. Mirrahimi and P. Rouchon, "Singular perturbations and Lindblad-Kossakowski differential equations," IEEE Transactions on Automatic Control, vol. 54, no. 6, pp. 1325-1329, 2009. 